\title{
Tendências climáticas e produção de uva na região dos Vales da Uva Goethe
}

\author{
Álvaro José Back ${ }^{(1)}$, Emilio Della Bruna ${ }^{(1)}$ e Hamilton Justino Vieira(2) \\ (1)Empresa de Pesquisa Agropecuária e Extensão Rural de Santa Catarina (Epagri), Rodovia SC 446, Km 16, Caixa Postal 49, \\ CEP 88840-000 Urussanga, SC. Email: ajb@epagri.sc.gov.br, emilio@epagri.sc.gov.br ${ }^{(2)}$ Epagri, Rodovia Admar Gonzaga, № 1.347, Itacorubi, \\ CEP 88034-901 Florianópolis, SC. Email: vieira@epagri.sc.gov.br
}

Resumo - O objetivo deste trabalho foi avaliar tendências nas séries climáticas e nos índices agroclimáticos para o cultivo da videira na região dos Vales da Uva Goethe, em Santa Catarina. A região apresenta clima mesotérmico úmido. Foram utilizados dados de temperatura máxima e mínima do ar, do período de 1924 a 2010, e de precipitação pluvial, de 1955 a 2010. O teste de Mann-Kendall foi utilizado para avaliar a tendência nas séries, cuja magnitude foi estimada pela declividade mediana determinada pelo teste de Theil-Sen. Foi observada tendência de aumento nas séries de temperatura mínima do ar, em escala anual, bem como nas estações do ano. Quanto à temperatura máxima, somente houve tendência de aumento na série de verão. Em relação aos índices agrometeorológicos, foi observada tendência de diminuição do número de geadas, diminuição do período entre a brotação e a colheita, aumento da soma térmica e dos índices de Huglin e de frio. Foram evidenciadas tendências de aumento da precipitação pluvial total anual e da precipitação no período de crescimento da videira, bem como aumento do número de dias, com chuva igual ou superior a $20 \mathrm{~mm}$, e aumento na temperatura mínima e noturna.

Termos para indexação: aquecimento global, climatologia, índice de frio, mudanças climáticas, precipitação pluvial, soma térmica.

\section{Climate trends and production of grapes in the Region of Goethe Grape Valleys, Brazil}

\begin{abstract}
The objective of this work was to evaluate the trends in climate series and in agroclimatic indices for growing vines in the Region of Goethe Grape Valleys, in the state of Santa Catarina, Brazil. The Region has a mesothermal humid climate. Maximum and minimum air temperatures, from the years 1924 to 2010, and rainfall data from 1955 to 2010 were used. The Mann-Kendall's test was used to evaluate the series trend, whose magnitude was estimated by the median slope determined by the Theil-Sen test. An increase trend was observed in the series of minimum air temperature, at the annual scale, as well as in the seasons of the year. For the maximum air temperature, there was a significant increase trend only in in the summer series. For the agrometeorological indices, a significant trend of decrease in the number of frost events was observed, reduction of the time between sprouting and harvesting, and increase trends in thermal summation and on the Huglin's and cold indexes. Increase trends were evidenced for the annual total rainfall and for rainfall during the vine growth period, as well as for the number of days with rain equal to or higher than $20 \mathrm{~mm}$, and for the minimum and night temperatures.
\end{abstract}

Index terms: global warming, climatology, cold index, global change, pluvial precipitation, thermal summation.

\section{Introdução}

As alterações climáticas projetadas pelos modelos climáticos mostram aumento na temperatura média do planeta (Solomon et al., 2007), em especial na região Sul do Brasil, e indicam aumento da ocorrência de chuvas (Marengo et al., 2009).

Segundo Tebaldi et al. (2006), umas das mais importantes implicações das mudanças climáticas são seus efeitos potenciais na variabilidade do clima e, consequentemente, nos eventos extremos, o que pode afetar de modo negativo as culturas agrícolas (Streck et al., 2011). Estudos que consideram séries temporais longas de elementos meteorológicos foram realizados em diferentes análises, que incluem a determinação de suas tendências (Kayano \& Sansigolo, 2009; Blain, 2011; Santos, 2011). Alexander et al. (2006) analisaram dados globais extremos de temperatura do ar e precipitação pluvial, na escala diária, e identificaram elevação significativa nas temperaturas noturnas, em $70 \%$ das regiões analisadas.

$\mathrm{O}$ aumento na temperatura pode ter impactos na produção da uva e causar modificação na área de cultivo e nas variedades cultivadas (Jones et al., 2005). Duchêne \& Schneider (2005) estudaram tendências de índices climáticos e agroclimáticos e a produção 
de uva na França. Nemani et al. (2001) afirmaram que as mudanças climáticas, observadas nos anos de 1951 a 1997, determinaram a diminuição da frequência de geadas e aumento da demanda evaporativa e duração do ciclo de videiras cultivadas na Califórnia. Muitas dessas modificações climáticas são vistas como vantajosas para a viticultura (Jones et al., 2005); no entanto, Nemani et al. (2001) salientam que elas podem não continuar a ser benéficas para a indústria vinícola, em razão da possibilidade de aumento de pragas e doenças. Segundo Jones et al. (2005), o aquecimento observado nos últimos 50 anos trouxe benefícios para muitas regiões vitícolas, tal como a redução de geadas. Estes benefícios são mais evidentes nas regiões de clima frio. Todavia, nas regiões vitícolas mais quentes e mais secas, houve necessidade de maiores investimentos em irrigação, o que aumentou os custos de produção.

Entre as centenas de variedades introduzidas e cultivadas na região dos Vales da Uva Goethe, apenas três atingiram áreas expressivas de cultivo, a 'Goethe', 'Bordô' e a 'Niagara'. Com o crescimento da população urbana da região, a partir de 1960, 'Niagara Rosada' passou a ser a principal cultivar plantada, e sua produção no sul do Estado de Santa Catarina foi direcionada ao consumo in natura.

O conhecimento dos impactos potenciais de alterações no clima sobre a viticultura é necessário, para que pesquisadores e produtores tomem decisões quanto a políticas e práticas de manejo que possam minimizar os impactos negativos e explorar os possíveis impactos positivos. Este trabalho teve como premissa o fato de que a viticultura, na região dos Vales da Uva Goethe, se desenvolve em clima quente, caracterizado por excessos hídricos e, portanto, pode ser seriamente impactada pelas mudanças climáticas.

O objetivo deste trabalho foi avaliar tendências nas séries climáticas e nos índices agroclimáticos para o cultivo da videira na região dos Vales da Uva Goethe, em Santa Catarina.

\section{Material e Métodos}

Foram utilizados os dados da Estação Meteorológica de Urussanga, $\mathrm{SC}\left(28^{\circ} 32^{\prime} \mathrm{S}, 49^{\circ} 19^{\prime} \mathrm{W}\right.$ e 49 m de altitude). Utilizaram-se dados mensais de temperaturas máxima e mínima do ar do período de 1924 a 2010, além de dados diários de temperaturas máxima e mínima do ar e de precipitação pluvial de 1955 a 2010 . O clima da região, pela classificação de Köppen, é mesotérmico úmido, com chuvas normalmente bem distribuídas e verão quente $(\mathrm{Cfa})$. A temperatura do mês mais frio varia de 13 a $15^{\circ} \mathrm{C}$, a média normal é de 17,0 a $19,3^{\circ} \mathrm{C}$, a média normal das máximas varia de 23,4 a $25,9^{\circ} \mathrm{C}$ e a das mínimas de 12,0 a $15,1^{\circ} \mathrm{C}$. A precipitação pluvial anual pode variar de 1.220 a $1.660 \mathrm{~mm}$.

Foram determinados os índices climáticos definidos pelo "Expert Team on Climate Change Detection, Monitoring and Indices" (ETCCDMI), conforme Peterson (2005), e os índices agroclimáticos aplicados à videira (Tabela 1). Estes índices foram calculados em escala anual, por estação do ano, e também na estação de crescimento da cultura da videira, de agosto a janeiro.

O impacto potencial da mudança climática na viticultura foi avaliado pela análise de tendência de índices agroclimáticos (Tabela 1), entre os quais: número de dias com geada (FD); data das fases fenológicas de brotação (GDDb), florescimento (GDDf) e colheita (GDDc), expressa em dia do ano; soma térmica de agosto a janeiro (STA10), com $10^{\circ} \mathrm{C}$ de temperatura basal; índice de frio (IF); índice de Huglin (IH); e número de dias com chuva superior a $20 \mathrm{~mm}$ no período vegetativo $\left(\mathrm{R} 20_{\mathrm{ec}}\right)$. Segundo Orlandini et al. (2009), esses índices são bons indicadores da interação entre mudanças no clima e necessidades fisiológicas.

A soma térmica foi calculada como STA10 $=\Sigma\left(T_{\text {méd }}-10\right)$, em que: STA10 é a soma térmica acima da $10^{\circ} \mathrm{C}$ (graus-dia); $\mathrm{T}_{\text {méd }}$ é a temperatura média diária $\left({ }^{\circ} \mathrm{C}\right)$, calculada como $\mathrm{T}_{\text {méd }}=\left(\mathrm{T}_{\mathrm{x}}+\mathrm{T}_{\mathrm{n}}\right) / 2$, em que $\mathrm{T}_{\mathrm{x}}$ é a temperatura máxima diária $\left({ }^{\circ} \mathrm{C}\right)$ e $\mathrm{T}_{\mathrm{n}}$ é a temperatura mínima diária $\left({ }^{\circ} \mathrm{C}\right)$.

$\mathrm{O}$ índice de Huglin $\left({ }^{\circ} \mathrm{C}\right)$ foi dado por $\mathrm{IH}=\Sigma\left[\left(\mathrm{T}_{\text {méd }}-\mathrm{T}_{\mathrm{b}}\right)+\left(\mathrm{T}_{\mathrm{x}}-\mathrm{T}_{\mathrm{b}}\right) / 2\right] \mathrm{K}$, em que: $\mathrm{T}_{\mathrm{b}}$ é a temperatura de base $\left(10^{\circ} \mathrm{C}\right)$; e $\mathrm{K}$ é um coeficiente multiplicador que, para a latitude da região em estudo, assume valor 1,0 (Conceição \& Tonnieto, 2005). No cálculo do $\mathrm{IH}$, foram usados os valores médios mensais multiplicados pelo número de dias do mês. Esse índice é usado na classificação de regiões vitícolas, na caracterização da adaptação da cultivar às condições climáticas, na caracterização de "terroirs" - uma extensão limitada de terra considerada do ponto de vista de suas aptidões agrícolas à produção vitícola, particularmente -, no monitoramento do desenvolvimento fisiológico e bioquímico da uva e

Pesq. agropec. bras., Brasília, v.47, n.4, p.497-504, abr. 2012 
na definição do estágio fenológico e data de colheita (Tonietto \& Carbonneau, 2004).

Para avaliar a tendência de mudanças climáticas, foi utilizado o teste não paramétrico de Mann-Kendall. Segundo Burn et al. (2004), as hipóteses a serem testadas são: H0, os dados são uma amostra de uma variável aleatória de $\mathrm{n}$ dados independentes e identicamente distribuídos, isto é, não existe tendência no conjunto de dados; H1, a distribuição dos dados xi e xj não é idêntica para todos os $i, j \leq N$, em que $i \neq$ onde i e $j$ são as sequências dos dados, e $\mathrm{N}$ é o comprimento da série. $\mathrm{Na}$ estatística Mann-Kendall, S é dado por:

$S=\sum_{j=1}^{N-1} \sum_{i=j+1}^{N} \operatorname{sgn}\left(x_{i}-x_{j}\right)$, em que sgn $(\theta)=\left\{\begin{array}{rr}1 & \text { se } \theta>0 \\ 0 & \text { se } \theta=0 \\ -1 & \text { se } \theta<0\end{array}\right\}$.

Para a variável aleatória independente distribuída identicamente (sem tendência), a média e a variância de $\mathrm{S}$ são dadas por $\mathrm{E}(\mathrm{S})=0$ e $\operatorname{Var}(\mathrm{S})=[\mathrm{N}(\mathrm{N}-1)$
$(2 \mathrm{~N}+5)-\Sigma(\mathrm{t}(\mathrm{t}-1)(2 \mathrm{t}+5)] / 18$, em que t é a extensão a qualquer empate.

Para o teste bicaudal, ao nível de significância $\alpha$, rejeita-se Ho se o teste estatístico padronizado apresentar valores $\mathrm{Z}<\mathrm{Z}_{\alpha / 2}$ ou $\mathrm{Z}>\mathrm{Z}_{\alpha / 2}$, em que a estatística normalizada $Z$ de Mann-Kendall segue a distribuição normal, com média zero e variância um. $\mathrm{O}$ valor de $\mathrm{Z}$ é calculado por

$$
Z=\left\{\begin{array}{l}
(S-1) / \sqrt{\operatorname{var}(S)} \text { se } S>0 \\
0 \quad \text { se } S=0 \\
(S+1) / \sqrt{\operatorname{var}(S)} \text { se } S<0
\end{array}\right\}
$$

De posse do valor de $\mathrm{Z}$, pode-se estimar o valor $\mathrm{p}$, que indica a quantidade de evidência para rejeitar Ho. Dessa forma, rejeitou-se a hipótese nula sempre que $\mathrm{p}$ foi menor que o nível de significância de 5\%.

O teste de Mann-Kendall não fornece estimativa da magnitude da tendência. Para obter essa estimativa, foi utilizado o teste não paramétrico de Theil-Sen (Helsel \& Hirsch, 2002), em que a estimativa da inclinação $(\beta)$ é

Tabela 1. Índices climáticos e agroclimáticos usados e suas definições.

\begin{tabular}{|c|c|c|}
\hline Índice & Unidade & Descrição \\
\hline $\mathrm{TNa}$ & ${ }^{\circ} \mathrm{C}$ & Média da Temperatura mínima anual \\
\hline $\mathrm{TNp}$ & ${ }^{\circ} \mathrm{C}$ & Média da Temperatura mínima diária na primavera (setembro novembro) \\
\hline $\mathrm{TNV}$ & ${ }^{\circ} \mathrm{C}$ & Média da Temperatura mínima diária no verão (dezembro fevereiro) \\
\hline TNo & ${ }^{\circ} \mathrm{C}$ & Média da Temperatura mínima diária no outono (março maio) \\
\hline TNi & ${ }^{\circ} \mathrm{C}$ & Média da Temperatura mínima diária no inverno (junho a agosto) \\
\hline TXa & ${ }^{\circ} \mathrm{C}$ & Média da Temperatura máxima anual \\
\hline $\mathrm{TXp}$ & ${ }^{\circ} \mathrm{C}$ & Média da Temperatura máxima diária na primavera (setembro novembro) \\
\hline TXv & ${ }^{\circ} \mathrm{C}$ & Média da Temperatura máxima diária no verão (dezembro fevereiro) \\
\hline TXo & ${ }^{\circ} \mathrm{C}$ & Média da Temperatura máxima diária no outono (março maio) \\
\hline TXi & ${ }^{\circ} \mathrm{C}$ & Média da Temperatura máxima diária no inverno (junho a agosto) \\
\hline TN90p & Dias & Índice de noites quentes: número de dias com temperatura mínima acima do percentil 90 (1961-1990). \\
\hline TX90p & Dias & Índice de dias quentes: número de dias com temperatura máxima acima do percentil 90 (1961-1990). \\
\hline FDa & Dias & Número de dias do ano com temperatura mínima abaixo de $0^{\circ} \mathrm{C}$ \\
\hline GDDb & Dias & $\begin{array}{l}\text { Data da brotação: dia do ano em que a soma da diferença entre a temperatura média diária e a temperatura base }\left(10^{\circ} \mathrm{C}\right) \text { alcança } \mathrm{o} \\
\text { valor específico }\end{array}$ \\
\hline GDDf & Dias & $\begin{array}{l}\text { Data do florescimento: dia do ano em que a soma da diferença entre a temperatura média diária e a temperatura base }\left(10^{\circ} \mathrm{C}\right) \text { alcança } \\
\text { o valor específico }\end{array}$ \\
\hline GDDc & Dias & $\begin{array}{l}\text { Data da colheita: dia do ano em que a soma da diferença entre a temperatura média diária e a temperatura base }\left(10^{\circ} \mathrm{C}\right) \text { alcança o valor } \\
\text { específico }\end{array}$ \\
\hline HI & Graus & $\begin{array}{l}\text { Índice de Huglin: soma diária da média entre a temperatura máxima e a média calculada durante a estação de crescimento (março a } \\
\text { setembro), multiplicada pelo coeficiente de latitude }\end{array}$ \\
\hline IF & ${ }^{\circ} \mathrm{C}$ & Índice de frio: representado pela temperatura mínima do mês antecedente à colheita (dezembro) \\
\hline STA10 & ${ }^{\circ} \mathrm{C}$ & Soma térmica (temperatura base $10^{\circ} \mathrm{C}$ ): soma da temperatura média diária do período de crescimento (março a setembro) \\
\hline $\mathrm{Pa}$ & $\mathrm{mm}$ & Precipitação pluvial total anual \\
\hline Pec & $\mathrm{mm}$ & Precipitação pluvial total durante a estação de crescimento (agosto a janeiro) \\
\hline Pv & $\mathrm{mm}$ & Precipitação pluvial total durante o verão \\
\hline $\mathrm{R} 20 \mathrm{v}$ & № & Número de dias com chuva superior a $20 \mathrm{~mm}$ durante o verão \\
\hline R20ec & № & Número de dias com chuva superior a $20 \mathrm{~mm}$ durante a estação de crescimento \\
\hline
\end{tabular}


obtida por $\beta=\operatorname{mediana}\left[\left(\mathrm{y}_{\mathrm{j}}-\mathrm{y}_{\mathrm{i}}\right) /\left(\mathrm{t}_{\mathrm{j}}-\mathrm{t}_{\mathrm{i}}\right)\right]$, para todos os $\mathrm{i}>\mathrm{j}$, em que, y é a variável testada para tendência e té o tempo; e $\beta$ representa a mediana da inclinação obtida de todas as possíveis combinações de dois pontos da série temporal.

\section{Resultados e Discussão}

Todos os índices de temperatura mínima apresentaram tendência de aumento (Tabela 2). A temperatura mínima anual $(\mathrm{TNa})$, com mediana $14,06^{\circ} \mathrm{C}$, apresentou inclinação positiva média $(\beta)$ de $0,0209^{\circ} \mathrm{C}$ por ano (Figura 1), o que indica aumento aproximado de $2^{\circ} \mathrm{C}$ por século. Maiores aumentos foram verificados no verão (TNv) $\left(\beta=0,0297^{\circ} \mathrm{C}\right.$ por ano) e primavera (TNpri) $\left(\beta=0,0255^{\circ} \mathrm{C}\right.$ por ano), épocas em que ocorre o desenvolvimento de grande parte do ciclo vegetativo da videira. Os menores aumentos foram observados no inverno $\left(\beta=0,0114^{\circ} \mathrm{C}\right.$ por ano).

Para a temperatura máxima anual, não foi identificada tendência (Figura 1) e, para as máximas nas estações,

Tabela 2. Resultados dos testes estatísticos de tendência dos índices climáticos, aplicados ao cultivo da videira em Urussanga, SC.

\begin{tabular}{|c|c|c|c|c|c|c|c|}
\hline \multirow[t]{2}{*}{ Índice ${ }^{(1)}$} & \multicolumn{3}{|c|}{ Teste de Mann-Kendal } & \multirow[t]{2}{*}{ Mediana } & \multicolumn{3}{|c|}{ Teste de Theil-Sen } \\
\hline & $\mathrm{S}$ & $\mathrm{Z}$ & $\mathrm{p}$ & & $\beta$ & $\beta$ inferior & $\beta$ superior \\
\hline $\mathrm{TNa}$ & 1624 & 6,054 & $<0,0001$ & 14,06 & 0,0209 & 0,0015 & 0,0275 \\
\hline $\mathrm{TNp}$ & 1392 & 5,188 & $<0,0001$ & 13,68 & 0,0255 & 0,0165 & 0,0333 \\
\hline $\mathrm{TNv}$ & 1761 & 6,565 & $<0,0001$ & 17,99 & 0,0297 & 0,0221 & 0,0367 \\
\hline TNo & 984 & 3,666 & 0,0002 & 14,84 & 0,0200 & 0,0102 & 0,0303 \\
\hline $\mathrm{TNi}$ & 1.827 & 2,119 & 0,0341 & 9,48 & 0,0114 & 0,0006 & 0,0217 \\
\hline TXa & 403 & 1,499 & 0,1339 & 26,05 & 0,0045 & $-0,0013$ & 0,0109 \\
\hline TXp & 542 & 2,018 & 0,0436 & 25,50 & 0,0090 & 0,0002 & 0,0167 \\
\hline TXv & 289 & 1,074 & 0,2828 & 29,83 & 0,0048 & $-0,0044$ & 0,0143 \\
\hline TXo & 408 & 1,518 & 0,1290 & 26,60 & 0,0059 & $-0,0022$ & 0,0146 \\
\hline TXi & 47 & 0,172 & 0,8634 & 22,61 & 0,0010 & $-0,0090$ & 0,0098 \\
\hline TN90p & 343 & 2,417 & 0,0156 & 8,50 & 0,1491 & 0,0266 & 0,2838 \\
\hline TX90p & -626 & $-4,417$ & 0,0000 & 6,00 & $-0,1559$ & $-0,2183$ & $-0,0930$ \\
\hline $\mathrm{FDa}$ & -422 & $-2,975$ & 0,0015 & 6,00 & $-0,0957$ & $-0,1496$ & $-0,0326$ \\
\hline GDDb & -268 & $-1,371$ & 0,0852 & 259,00 & $-0,0909$ & $-0,1852$ & 0,0000 \\
\hline GDDf & -160 & $-1,124$ & 0,1305 & 289,00 & $-0,0589$ & $-0,1429$ & 0,0306 \\
\hline GDDc & -377 & $-2,657$ & 0,0039 & 377,00 & $-0,1429$ & $-0,2500$ & 0,0349 \\
\hline HI & 953 & 3,551 & 0,0004 & $2.654,90$ & 1,8493 & 0,9684 & 2,8593 \\
\hline IF & 1.448 & 5,305 & $<0,0001$ & 17,07 & 0,0329 & 0,0223 & 0,0432 \\
\hline STA10 & 612 & 4,318 & $<0,0001$ & $2.743,20$ & 5,8040 & 3,3710 & 8,0870 \\
\hline $\mathrm{Pa}$ & 442 & 3,117 & 0,0018 & $1.081,90$ & 7,8270 & 3,2100 & 12,3880 \\
\hline Pec & 325 & 2,290 & 0,0220 & 549,40 & 3,4190 & 0,6650 & 6,0970 \\
\hline $\mathrm{Pv}$ & 306 & 2,155 & 0,0312 & 829,80 & 4,2350 & 0,3795 & 8,0872 \\
\hline $\mathrm{R} 20 \mathrm{v}$ & 424 & 2,990 & 0,0028 & 9,00 & 0,0870 & 0,0266 & 0,1579 \\
\hline $\mathrm{R} 20 \mathrm{ec}$ & 494 & 3,484 & 0,0005 & 14,00 & 0,1370 & 0,0588 & 0,2054 \\
\hline
\end{tabular}

${ }^{(1)}$ S, estatística de Mann-Kendall; Z, estatística normalizada de Mann-Kendall; $\beta$ mediana da estimativa de inclinação obtida de todas as possíveis combinações entre dois pontos da série temporal. foi verificada tendência para os valores de máxima na primavera, com aumento de $0,0090^{\circ} \mathrm{C}$ por ano. Este resultado está de acordo com as observações de Back (2001), que analisou dados de temperatura em Urussanga, SC, do período de 1924 a 1998, tendo aplicado os testes de Mann-Kendal, e observou tendência de aumento nas temperaturas média e mínima anuais, porém não observou tendência significativa na máxima anual. Vários trabalhos mostram que tem ocorrido aumento mais acentuado da temperatura mínima do que da temperatura máxima (Nemani et al., 2001; Vicent et al., 2005; Alexander et al., 2006; Blain et al., 2009; Kayano \& Sansigolo, 2009; Blain, 2011). Com o aumento da mínima, espera-se diminuição da frequência de geadas e aumento da estação de crescimento livre de geadas, fatores considerados como vantagens para a viticultura.

Quanto às temperaturas extremas, observou-se, nas séries analisadas, aumento significativo da frequência de dias com mínimas acima do percentil 90 (TN90p), o que indica maior ocorrências de noites quentes (Figura 1). Também houve tendência de redução da frequência de dias com temperaturas máximas acima do percentil 90 (TX90p), o que indica menor frequência de dias quentes. Grace et al. (2009) comentam que a qualidade da uva é afetada pelas altas temperaturas. Temperaturas extremas acima de $35^{\circ} \mathrm{C}$, registradas no abrigo meteorológico, que correspondem a temperaturas acima de $40^{\circ} \mathrm{C}$ sob o sol, são consideradas temperaturas prejudiciais para a videira, por que inibem ou mesmo bloqueiam processos fisiológicos e bioquímicos.

$\mathrm{O}$ número de geadas por ano (FD), com valor da mediana de seis geadas anuais, apresentou tendência de diminuição, com $\beta=-0,0957$ geadas por ano, o que indica, em média, diminuição de um dia com geada a cada década (Figura 2).

Com relação à duração das fases fenológicas, identificou-se tendência de antecipação da data da colheita (GDDc), com $\beta=-0,1429$ dias por ano. Para as fases de brotação (GDDb) e florescimento (GDDf), não houve tendência significativa. A soma térmica apresentou tendência de aumento $\left(\beta=5,8^{\circ} \mathrm{C}\right.$ por ano) (Figura 2), o que justifica a antecipação da data de colheita. Segundo Santos et al. (2009), a soma térmica é um bom indicador inicial da influência climática sobre um vinhedo em particular, mas pode não ser suficiente para descrever como o microclima influencia a maturação. A média diurna da temperatura 

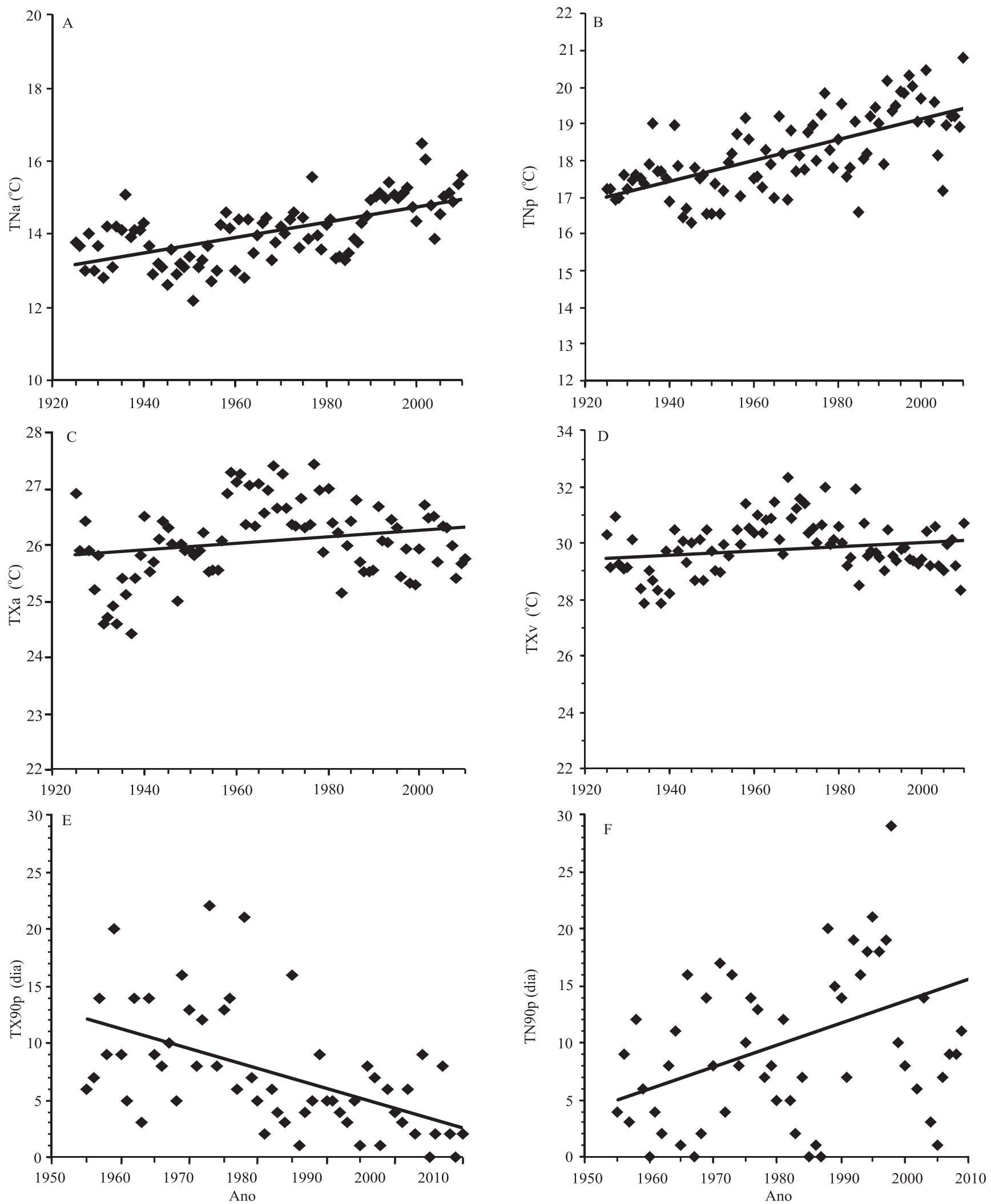

Figura 1. Tendência das séries: A, temperatura mínima anual (TNa); B, temperatura mínima na primavera $(\mathrm{TNp})$; C, temperatura máxima anual (TXa); D, temperatura máxima no verão TXv; E, número de dias quentes TX90p e F, número de noites quentes (TN90p). 

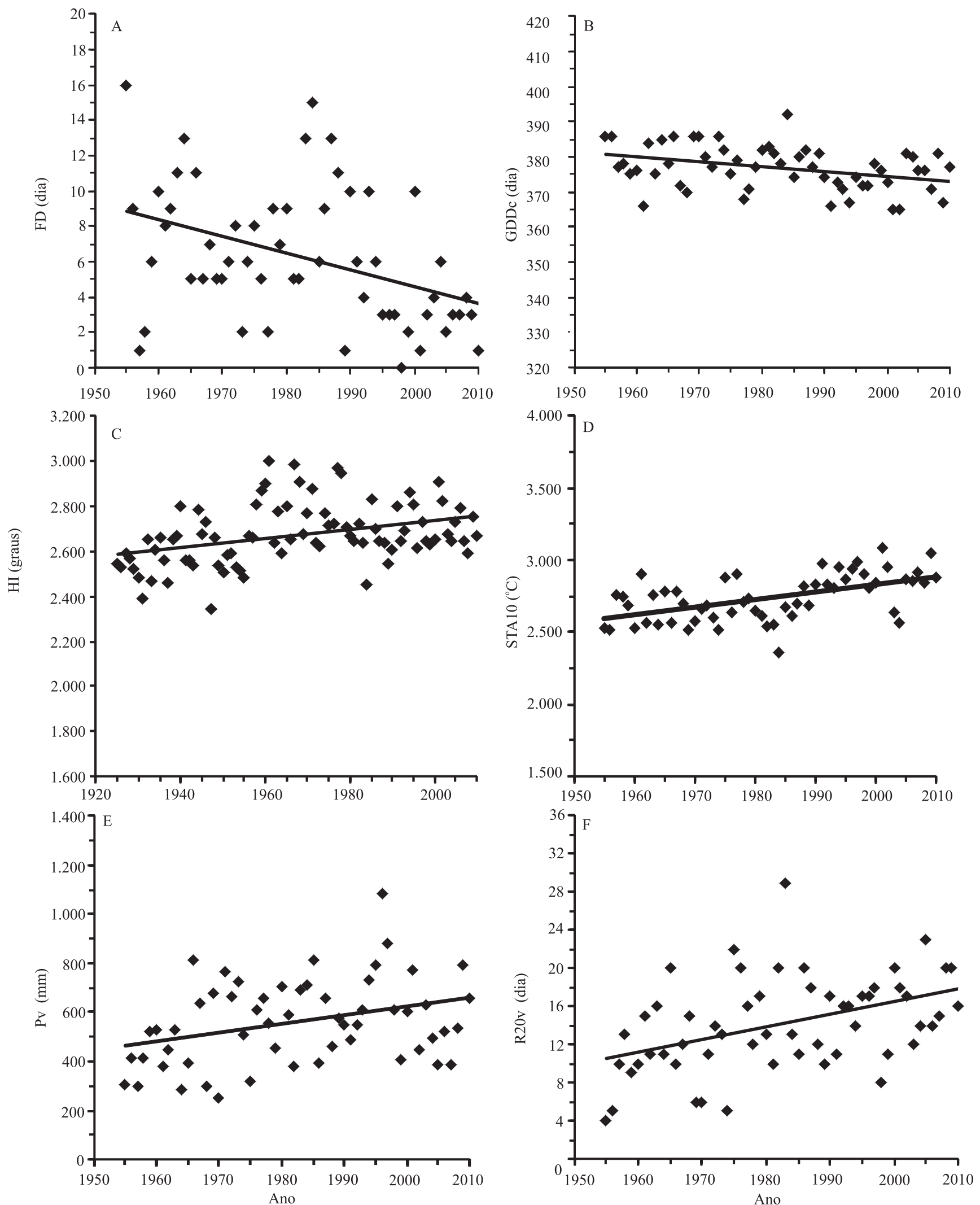

Figura 2. Tendência das séries: A, número de geadas anuais (FD) ; B, data da colheita (GDDc); C, índice de Huglin (HI); D, soma térmica (STA10); E, precipitação no verão (Pv); F, dias de chuva acima de $20 \mathrm{~mm}$ no verão (R20v). 
do ar durante a maturação é útil para este propósito. A diferença entre a temperatura do ar diurna e noturna, assim como as temperaturas mínimas nos dois períodos, esclarecem as condições para a maturação de um vinhedo em um local particular. De acordo com Santos et al. (2009), um clima com dias quentes e noites quentes produz um estilo de vinho diferente, em comparação com uma região com a mesma soma térmica, porém com dias quentes e noites frias.

$\mathrm{O}$ índice de Huglin mostrou tendência de aumento, $\operatorname{com} \beta=1,849^{\circ} \mathrm{C}$ por ano (Figura 2). Carey \& Bonnardot (2004) mostraram que há relações entre o IH e o aroma do vinho. A mediana do IH calculado foi de 2.654, o que caracteriza a região dos Vales da Uva Goethe como quente (Tonietto \& Carbonneau, 2004).

O índice de frio mediano foi de $17,07^{\circ} \mathrm{C}$, com tendência de aumento $\left(\beta=0,0329^{\circ} \mathrm{C}\right.$ por ano). De acordo com Conceição \& Tonnieto (2005), o valor de IF de $17,07^{\circ} \mathrm{C}$ caracteriza a região como classe de noites temperadas $(14<\mathrm{IF} \leq 18)$, mas, com a tendência de aumento, ela poderá passar para a classe de noites quentes, IF $>18$. $\mathrm{O}$ IF pode estar indiretamente relacionado à intensidade do aroma do vinho e à coloração do suco de variedades rosadas (Carbonneau et al., 2007). Em condições de maturação nictotérmicas quentes, a tendência é que se verifique perda de aromas e coloração relativamente fraca, enquanto condições de maturação nictotérmicas frias são essencialmente favoráveis à coloração e aos aromas das uvas.

Com relação à precipitação, os índices mostraram aumento significativo de chuva anual $(\beta=7,827 \mathrm{~mm}$ por ano), chuva durante a estação de crescimento ( $\beta=3,419 \mathrm{~mm}$ por ano) e, também, de chuva acumulada no verão ( $\beta=4,235 \mathrm{~mm}$ por ano) (Figura 2 ). Quanto aos dias de chuva acima de $20 \mathrm{~mm}$, observou-se tendência de aumento no verão ( $\beta=0,087$ chuvas por ano) e durante a estação de crescimento ( $\beta=0,137$ chuvas por ano). Estes dados estão de acordo com as observações de Back (2001), que identificou tendência significativa no aumento da chuva anual de Urussanga, SC, e encontrou, na análise por trimestre, alterações significativas somente na precipitação do trimestre outubro a dezembro.

A cultura da videira tem sua produção e qualidade afetadas tanto pelo excesso como pelo deficit hídrico. A água é um elemento fundamental para o crescimento vegetativo e reprodutivo da videira e para seu funcionamento fisiológico e bioquímico. Além disso, ela é fator determinante no rendimento e qualidade das uvas e vinhos (Ojeda et al., 2002; Deloire et al., 2005). $\mathrm{O}$ excesso hídrico, combinado a temperaturas elevadas, torna a cultura da videira muito suscetível a doenças fúngicas e a pragas.

Back \& Dela Bruna (2009) estimaram a demanda hídrica da videira de $335 \mathrm{~mm}$, na região de Urussanga. A precipitação mediana, durante o ciclo vegetativo, foi de $549 \mathrm{~mm}$, o que supera a demanda em 64\%. Estes dados estão de acordo com as observações de Back \& Dela Bruna (2009) de que, na região dos Vales da Uva Goethe, os excessos hídricos são mais frequentes que os déficits hídricos. A tendência de aumento da quantidade e da frequência de chuvas, durante o período vegetativo, poderá favorecer o aparecimento de doenças e aumentar a necessidade de pulverizações. Bardin et al. (2010), por exemplo, usaram o critério da pulverização após chuva de $20 \mathrm{~mm}$ para determinar o risco de ocorrência de doenças fúngicas na videira 'Niágara Rosada' no Estado de São Paulo. Pode-se inferir, pelo índice R20 no período vegetativo, um aumento do número de pulverizações contra doenças fúngicas, com consequente aumento dos custos e diminuição da qualidade dos frutos.

\section{Conclusões}

1. Há tendência de aumento da temperatura mínima anual em todas as estações do ano, com maior valor no verão.

2. A temperatura máxima anual apresenta tendência significativa de aumento somente no período da primavera.

3. Há tendência de diminuição do número de geadas, aumento da soma térmica e aumento do índice de Huglin, além de diminuição do período entre a brotação e a maturação.

4. Há tendência de aumento da chuva total anual, bem como da chuva no período vegetativo e do número de dias de chuva acima de $20 \mathrm{~mm}$.

\section{Referências}

ALEXANDER, L.V.; ZHANG, X.; PETERSON, T.C.; CAESAR, J.; GLEASON, B.; KLEIN TANK, A.M.G.; HAYLOCK, M.; COLLINS, D.; TREWIN, B.; RAHIMZADEH, F.; TAGIPOUR, A.; RUPA KUMAR, K.; REVEDEKAR, J.; GRIFFITHS, G.; VICENT, L.; STEPHENSON, D.B.; BURN, J.; AGUILAR, E.; BRUNET, M.; TAYLOR, M.; NEW, M.; ZHAI, P.; RUSTICUCCI, M.; VAZQUEZ-AGUIRRE, J.L. Global observed changes in daily climate extremes of temperature and precipitation. Journal of Geophysical Research, v.111, 2006. Doi:10.1029/2005JD006290. 
BACK, Á.J. Aplicação de análise estatística para identificação de tendências climáticas. Pesquisa Agropecuária Brasileira, v.36, p.717-726, 2001.

BACK, Á.J.; DELLA BRUNA, E. Demanda hídrica e necessidade de irrigação da videira para Urussanga, SC. Agropecuária Catarinense, v.22, p.76-81, 2009.

BARDIN, L.; PEDRO JÚNIOR, M.J.; MORAES, J.F.L. de. Risco climático de ocorrência de doenças fúngicas na videira 'Niágara Rosada' na região do polo turístico do circuito das frutas do Estado de São Paulo. Bragantia, v.69, p.1019-1026, 2010.

BLAIN, G.C. Considerações estatísticas relativas a seis séries mensais de temperatura do ar da Secretaria de Agricultura e Abastecimento do Estado de São Paulo. Revista Brasileira de Meteorologia, v.26, p.279-296, 2011.

BLAIN, G.C.; PICOLE, M.C.A.; LULU, J. Análises estatísticas das tendências de elevação nas séries de temperatura mínima do ar no Estado de São Paulo. Bragantia, v.68, p.807-815, 2009.

BURN, D.H.; CUNDERLIK, J.M.; PIETRONIRO, A. Hydrological trends and variability in the Liard River basin. Hydrological Sciences Journal, v.49, p.53-68, 2004.

CARBONNEAU, A.; DELOIRE, A.; JAILLARD, B. La vigne: physiologie, terroir, culture. Paris: Dunod, 2007. 441p.

CAREY,V.A.;BONNARDOT,V.Aviticultural perspectiveofmeso-scale atmospheric modelling in the Bottelaryberg-Simonsberg-Helderberg wine growing area (South Africa). Bulletin de l'Office International de la Vigne et du Vin, v.77, p.20-46, 2004.

CONCEIÇÃO, M.A.F.; TONIETTO, J. Climatic potential for wine grape production in the tropical north region of Minas Gerais State, Brazil. Revista Brasileira de Fruticultura, v.37, p.404-407, 2005.

DELOIRE, A.; OJEDA, H.; ZEBIC, O.; BERNARD, N.; HUNTER, J.J.; CARBONNEAU, A. Influence de l'état hydrique de la vigne sur le style de vin. Progrès Agricole et Viticole, v.122, p.455-462, 2005.

DUCHÊNE, E.; SCHNEIDER, C. Grapevine and climatic changes: a glance at the situation in Alsace. Agronomy for Sustainable Development, v.25, p.93-99, 2005.

GRACE, W.J.; SANDRAS, V.O.; HAYMAN, P.T. Modelling heatwaves in viticultural regions of Southeastern Australia. Australian Meteorological and Oceanographic Journal, v.58, p.249-262, 2009.

HELSEL, D.R.; HIRSCH, R.M. Statistical methods in water resources. In: TECHNIQUES of water-resources investigations of the United States Geological Survey. Washington: U.S. Government Printing Office, 2002. chap. A3, 510p. (Book 4. Hydrologic analysis and interpretation).

JONES, G.V.; WHITE, M.A.; COOPER, O.R.; STORCHMANN, K. Climate Change and global wine quality. Climate Change, v.73, p.319-343, 2005.

KAYANO, M.T.; SANSIGOLO, C. Interannual to decadal variations of precipitation and daily maximum and daily minimum temperatures in Southern Brazil. Theoretical an Applied Climatology, v.97, p.81-90, 2009.
MARENGO, J.A.; JONES, R.; ALVES, L.M.; VALVERDE, M.C. Future change of temperature and precipitation extremes in South America as derived from the PRECIS regional climate modeling system. International Journal of Climatology, v.29, p.2241-2255, 2009.

NEMANI, R.R.; WHITE, M.A.; CAYAN, D.R.; JONES, G.V.; RUNNING, S.W.; COUGHLAN, J.C.; PETERSON, D.L. Asymmetric warming over coastal California and its impact on the premium wine industry. Climate research, v.19, p.25-34, 2001.

OJEDA, H.; ANDARY, C.; KRAEVA, E.; CARBONNEAU, A.; DELOIRE, A. Influence of pre- and postveraison water deficit on synthesis and concentration of skin phenolic compounds during berry growth of Vitis vinifera cv. Shiraz. American Journal of Viticulture and Enology, v.53, p.261-267, 2002

ORLANDINI, S.; DI STEFANO, V.; LUCCHESINI, P.; PUGLISI, A.; BARTOLINI, G. Current trends of agroclimatic indices applied to grapevine in Tuscany (Central Italy). Idojaras, v.113, p.69-78, 2009.

PETERSON, T.C. Climate change indices. World meteorological Organization Bulletin, v.54, p.83-86, 2005.

SANTOS, A.O.; ROLIM, G. de S.; HERNANDES, J.L.; PEDRO JÚNIOR, M.J. A maturação fisiológica da videira vinífera em São Paulo: comentários sobre as safras de verão e de inverno na média altitude paulista. 2009. Disponível em: <http://www.infobibos.com/ Artigos/2009_3/maturacao/index.htm>. Acesso em: 23 abr. 2012.

SANTOS, C.A.C. dos. Trends in indices for extremes in daily air temperature over Utah, USA. Revista Brasileira de Meteorologia, v.26, p.19-28, 2011.

SOLOMON, S.; QIN, D.; MANNING, M.; CHEN, Z.; MARQUIS, M.; AVERYT, K.B.; TIGNOR, M.; MILLER, H.L. (Ed.). Climate change 2007: the physical science basis: contribution of Working Group I to the Fourth Assessment Report of the Intergovernmental Panel on Climate Change. Cambridge: Cambridge University, 2007. 996p.

STRECK, N.A.; GABRIEL, L.F.; BURIOL, G.A.; HEDWEIN, A.B.; PAULA, G.M. Variabilidade interdecadal na série secular de temperatura do ar em Santa Maria, RS. Pesquisa Agropecuária Brasileira, v.46, p.781-790, 2011.

TEBALDI, C.; HAYHOE, K.; ARBLASTER, J.M.; MEEHL, G.A. Going to the extremes: an intercomparison of model-simulated historical and future changes in extreme events. Climate Change, v.79, p.185-211, 2006.

TONIETTO, J.; CARBONNEAU, A. A multicriteria climatic classification system for grape-growing regions worldwide. Agricultural and Forest Meteorology, v.124, p.81-97, 2004.

VICENT, L.A.; PETERSON, T.C.; BARROS, V.R.; MARINO, M.B.; RUSTICUCCI, M.; CARRASCO, G.; RAMIREZ, E.; ALVES, L.M.; AMBRIZZI, T.; BERLATO, M.A.; GRIMM, A.M.; MARENGO, J.A.; MOLION, L.; MONCUNILL, D.F.; REBELLO, E.; ANUNCIAÇÃO, Y.M.T.; QUINTANA, J.; SANTOS, J.L.; BAEZ, J.; CORONEL, G.; GARCIA, J.; TREBEJO, I.; BIDEGAIN, M.; HAYLOCK, M.R.; KAROLY, D. Observed trends in indices of daily temperature extremes in South America 1960-2000. Journal of Climate, v.18, p.5011-5023, 2005.

Recebido em 15 de dezembro de 2011 e aprovado em 16 de março de 2012

Pesq. agropec. bras., Brasília, v.47, n.4, p.497-504, abr. 2012 